\title{
Correlación de protoporfirina zinc y plomo en sangre en trabajadores de fábricas de baterías, de Bogotá, Colombia
}

\author{
0 mayda C árdenas-Bustamante, Bact., ${ }^{(1)}$ Marcela Eugenia Varona-U ribe, M.D.,(1) \\ Sandra Marcela N úñez-Trujillo, M.D., M.Sc., ${ }^{(1)}$ Jaime Eduardo 0 rtiz-Varón, Q uím., ${ }^{(1)}$
}

Germán E. Peña-Parra, M.D.(1)

\begin{abstract}
Cárdenas-Bustamante 0,Varona-Uribe ME, Núñez-Trujillo SM, Ortiz-Varón JE, Peña-Parra GE. Correlación de protoporfirina zinc y plomo en sangre en trabajadores de fábricas de baterías, de Bogotá, Colombia. Salud Publica Mex 2001;43:203-210. El texto completo en inglés de este artículo está disponible en: http://www.insp.mx/salud/index.html
\end{abstract}

\section{Resumen}

Objetivo. Determinar la utilidad de la protoporfirina zinc en sangre (PPz) como indicador de exposición a plomo en trabajado res de fábricas de baterías. Material y métodos. Se hizo un estudio transversal en 116 trabajadores de fábricas de baterías del sector informal en Bogotá, C olombia. La información sobre variables generales, ocupacionales y de salud fue obtenida por medio de una entrevista con los trabajadores. Se establecieron dos categorías para los valores de PPz: aquellos por debajo del valor límite de $(70 \mu \mathrm{g} / \mathrm{dl})$, y aquellos por arriba de este valor. Se empleó análisis de regresión lineal para medir la correlación entre los valores logarítmicos de PPz (>70 $\mu \mathrm{g} / \mathrm{dl})$ y plomo en sangre (PbS) (>38 $\mu \mathrm{g} / \mathrm{dl}$ ). Resultados. Se encontró un coeficiente de correlación semilo garítmica $r=0.54$, además de posibles asociaciones estadísticamente significativas entre los niveles elevados de PPz y el oficio actual de exposición directa (RM:3.35, IC 95\% 1.02-11.91; p:0.02), emplear plomo como materia prima (RM:7.80, IC 95\% 2.96-21.03; $p<0.01$ ) y cambio poco frecuente de ropa de trabajo (RM:3.55, IC $95 \%$ 1.17-11.01; $p<0.01$ ). Conclusiones. La PPz puede ser un buen indicador diagnóstico de intoxicación por plomo, y puede ser usada como prueba tamiz para apoyar los programas de vigilancia y seguimiento en el monitoreo biológico en trabajadores expuestos a plomo. El texto completo en inglés de este artículo está disponible en: http:// www.insp. $\mathrm{mx} / \mathrm{salud} / \mathrm{index}$.html

Palabras clave: protoporfirina zinc; plomo en sangre; plomo inorgánico; exposición ocupacional; Colombia

\author{
Cárdenas-Bustamante 0,Varona-Uribe ME, \\ Núñez-Trujillo SM, Ortiz-Varón JE, Peña-Parra GE. \\ Correlation of zinc protoporphyrin \\ with blood lead levels in car battery industry \\ workers in Bogota, Colombia. \\ Salud Publica Mex 2001;43:203-210. \\ The English version of this paper \\ is available at: http://www.insp.mx/salud/index.html
}

\begin{abstract}
A bstract
Objective To determine the usefulness of zinc protoporphyrin in blood (PPz) as an indicator of lead exposure in workers of the homemade car battery industry. Material and methods A cross-sectional study was performed in 116 workers of the car battery industry in Bogotá, Colombia. Data on general, occupational, and health variables were collected by interview. Two categories of PPz values were established:Those below the cutoff value $(70 \mu \mathrm{g} /$ $\mathrm{dL}$ ) and those above it. A linear regression analysis was performed to measure the correlation between lo garithm values of PPz $(>70 \mu \mathrm{g} / \mathrm{dL})$ and lead in blood (PbB) $(>38 \mu \mathrm{g} / \mathrm{dL})$. Results. A semi-logarithmic correlation coefficient of $r=0.54$ was found, and statistically significant associations between high levels of PPz and direct exposure to lead were observed (OR:3.35, 95\% IC 1.02-11.91; p:0.02); for workers who often use lead as a raw material (OR:7.80, $95 \%$ IC $2.96-21.03 ; p<0.01$ ), as well as for workers who do not change work clothes often (O R:3.55,95\% IC 1.17-11.01; $\mathrm{p}<0.01$ ). Conclusions. PPz may be a useful diagnostic indicator for lead poisoning; it may also be used as a screening test for surveillance programs in the biological monitoring of workers exposed to lead.The English version of this paper is available at:http://www.insp.mx/salud/index.html
\end{abstract}

Key words: protoporphyrin zinc; blood lead; inorganic lead; occupational exposure; Colombia

(1) Laboratorio Salud A mbiental, Instituto N acional de Salud, Bogotá, Colombia.

Fecha de recibido: 17 de marzo de 2000 - Fecha de aprobado: 14 de febrero de 2001

Solicitud de sobretiros: Bact. 0 mayda Cárdenas Bustamante. Laboratorio Salud Ambiental, Instituto $\mathrm{N}$ acional de Salud. Avenida El Dorado, Carrera 50, CAN , Zona 6 Bogotá, Colombia.

Correo electrónico: ocardenas@ hemagogus.ins.gov.co 
E 1 riesgo de exposición al plomo es muy distinto según se está en el ambiente general o en el ocupacional, ${ }^{1}$ donde la vía de absorción más importante es la respiratoria. ${ }^{1,2-4}$ El plomo absorbido se transporta por la sangre y una vez allí establece un rápido equilibrio entre eritrocitos y plasma, se distribuye en diversos órganos y tejidos, y se deposita principalmente en los huesos. ${ }^{1,4-7}$ El plomo interfiere en la síntesis de globulina en los eritrocitos, bloquea la ferroquelatasa impidiendo la unión del hierro a la protoporfirina IX para formar el grupo hemo, por lo que la protoporfirina IX se une al zinc, y forma la protoporfirina zinc $(\mathrm{PPz})$, lo cual incrementa la concentración de dicha metaloporfirina dentro del glóbulo rojo y disminuye la de la hemoglobina, hecho que produce anemia normocítica e hipocrómica y aumento en la concentración de hierro sérico. ${ }^{8-13}$

En Colombia no se tienen datos estadísticos ni se ha evaluado suficientemente la magnitud real del problema de salud derivado de la exposición ocupacional al plomo en el sector informal. El saturnismo afecta a $35 \%$ de quienes trabajan en fábricas de baterías, y a $14.6 \%$ de quienes lo hacen en fundición, imprentas y con cerámica. ${ }^{14,15}$ Varona encontró niveles de plomo en sangre $(\mathrm{PbS})$ por arriba de $66 \mu \mathrm{g} / \mathrm{dl}$ en $51 \%$ de los trabajadores. ${ }^{16}$

El proceso de reciclaje y fabricación de baterías en el sector informal del país utiliza el plomo metálico en la fundición de rejillas, bornes y soldadura, procesos donde todas las labores que se realizan son manuales y producen grandes desprendimientos de partículas de plomo metálico o de sus óxidos, ${ }^{16}$ que ocasionan graves intoxicaciones y contaminación ambiental.

Las fábricas participantes en el estudio fueron del sector informal de la economía (microempresas familiares), donde todos los trabajadores rotan por los diferentes oficios durante los procesos para reciclaje y fabricación de las baterías; aquéllos se inician con la compra de baterías viejas de las cuales reciclan y recuperan todas las piezas de plomo (bornes, puentes, rejillas, placas positivas y negativas), para posteriormente fundirse a temperaturas de $400-450{ }^{\circ} \mathrm{C}$ en estufas abiertas y en condiciones locativas muy precarias -no existe ventilación adecuada, debido a que los lugares de trabajo son totalmente cerrados. Este proceso se convierte en fuente importante de exposición a vapores, partículas y residuos de plomo inorgánico; si a esto se agrega el bajo porcentaje en el uso de elementos de protección personal y las malas condiciones de higiene, el riesgo de exposición a este metal puede ser mayor. Dicho problema no sólo afecta a los trabajadores; es, asimismo, una fuente de contaminación para los miembros de su familia. Una vez que se obtiene el plomo fundido se envasa en moldes de acero y aluminio reforzado, para la fabricación de las piezas con las cuales ensamblan las nuevas baterías.

Con base en la problemática anterior, y debido a que no existen fuentes de información que permitan evaluar el alcance del problema en la población del sector informal, se realizó este estudio con el fin de establecer un diagnóstico del estado actual de exposición a plomo, y determinar, de esta forma, si la $\mathrm{PPz}$ puede ser utilizada como prueba de tamizaje en los programas de vigilancia epidemiológica de trabajadores con niveles de PbS altos, en fábricas de baterías del sector informal en Bogotá.

\section{Material y métodos}

En 1996 se realizó este estudio transversal en 116 trabajadores de 12 fábricas de baterías que participaron voluntariamente, de un total de 20 fábricas contactadas del sector informal en Bogotá. Se estudió la totalidad de los trabajadores de las 12 empresas participantes, los expuestos directamente -aquellos que manipulaban el plomo en los procesos laborales- y el personal administrativo -quienes se encontraban indirectamente expuestos. Se hizo una prueba piloto en $10 \%$ del total de los trabajadores de la muestra, los cuales no formaron parte del estudio.

Antes de iniciar la recolección de la información y de las muestras biológicas, se informó a las empresas y a los trabajadores sobre los objetivos y el tipo de estudio que se desarrollaría. Una vez que aceptaron participar voluntariamente y firmaron un consentimiento escrito, se les hizo una entrevista para aplicarles un cuestionario para la recolección de información demográfica, antecedentes ocupacionales, clínicos y toxicológicos.

Para el análisis de PPz y PbS en un sitio aislado del lugar de trabajo, se tomó una muestra de $5 \mathrm{ml}$ de sangre, previa desinfección del área de venopunción. ${ }^{17}$ Las muestras se recolectaron en tubos vacutainer ${ }^{\circledR}$ que contenían anticoagulante salpotásica del ácido etilen diamino tetra acético (EDTA) y que estaban libres de metales pesados; éstas permanecieron refrigeradas hasta su análisis en el Laboratorio Salud Ambiental del Instituto Nacional de Salud de Colombia (INS).

La determinación de la PPz se realizó por hematofluorometría, ${ }^{18}$ y se utilizó un hematofluorómetro AVIV modelo 206 de la compañía Biomedical;'19 asimismo, se emplearon controles AVIV certificados de alta, media y baja concentración como controles de calidad internos para calibrar el equipo. El PbS se analizó por espectrofotometría de absorción atómica por horno 
de grafito, ${ }^{20}$ y se empleó un espectrofotómetro Perkin Elmer modelo 2380, horno de grafito HGA 400, con corrector de lámpara de deuterio, según el método de Lefebvre M. y colaboradores modificado en el Laboratorio Salud Ambiental del INS..$^{21} \mathrm{El}$ proceso analítico de plomo en sangre se hizo incluyendo muestras de referencia procedentes del Centro Toxicológico de Quebec, Canadá, por cada lote de muestras procesadas; con este centro, el Laboratorio Salud Ambiental viene participando desde 1987 en los Programas de Control de Calidad Internacional.

Para efectos de comparación de las variables $\mathrm{PPz}$ y $\mathrm{PbS}$, éstas se dicotomizaron con base en los límites aceptados para la población colombiana, ${ }^{22}$ en estudios realizados en el país ${ }^{15,18,23,24}$ y teniendo en cuenta lo reportado en la literatura. ${ }^{3}$ Con base en lo anterior, para PPz se tomaron como "valores anormales" los mayores a $70 \mu \mathrm{g} / \mathrm{dl}$, y como "valores normales", los menores o iguales a $70 \mu \mathrm{g} / \mathrm{dl}{ }^{15,18}$ Para PbS se tomaron como valores para población expuesta los mayores de $38 \mu \mathrm{g} / \mathrm{dl}$, y para población no expuesta, los menores o iguales a $38 \mu \mathrm{g} / \mathrm{dl}_{,}{ }^{3}$ valores límites permisibles fijados por el Ministerio de Salud de Colombia -por conducto del INS. 22,25

Se hizo análisis univariado a todas las variables para conocer las estadísticas descriptivas como medidas de tendencia central y dispersión. Algunas variables continuas se utilizaron en su escala de distribución original, y otras se dicotomizaron, como la variable nivel de $\mathrm{PPz}$, de acuerdo con lo definido en el estudio. Se determinaron las distribuciones simples para cada variable, así como los intervalos de confianza de $95 \%$.

Para establecer la relación entre los niveles de $\mathrm{PPz}$ y los de $\mathrm{PbS}$ se usó un modelo de regresión lineal. ${ }^{911,13,26}$ Inicialmente se realizó una correlación con los niveles de PPz en unidades originales, y posteriormente entre los valores logarítmicos de $\mathrm{PPz}(>70 \mu \mathrm{g} / \mathrm{dl})$ y los valores de $\mathrm{PbS}(>38 \mu \mathrm{g} / \mathrm{dl})$, establecidos como puntos de corte.

Las comparaciones entre los que presentan valores por encima y por debajo de los puntos de corte se hicieron en tablas de cuatro casillas para cada variable de exposición, utilizando como medida de asociación la razón de momios (RM), con sus intervalos de confianza de $95 \%$. Los tipos de oficio se codificaron para establecer una variable ordinal de dos categorías: oficios de exposición directa y de exposición indirecta al plomo. Las características demográficas, variables ocupacionales y clínicas se relacionaron con las concentraciones halladas de PPz en sangre, con el fin de obtener datos acerca de la población expuesta al plomo.
Se hizo el análisis de varianza (ANOVA) entre las medias de la variable nivel de PPz y las características del trabajador; para ello se utilizó la prueba estadística F con sus correspondientes valores de $p$, con el fin de identificar diferencias estadísticamente significativas. Por último, se realizó un análisis estratificado (por el método de Mantel y Haenszel) para comprobar que ninguna de las posibles asociaciones encontradas fuera el resultado de la interacción de otras variables. Este estudio no pretende establecer causalidad sino, a partir de los resultados, formular hipótesis de asociación que se evaluarán posteriormente mediante estudios analíticos. Para el análisis se utilizaron los programas Epi-Info 6.04 y Excel.

\section{Resultados}

Participaron en el estudio 116 trabajadores; 88.8\% (103) eran hombres y $11.2 \%$ (13) mujeres, con un promedio de edad de 32 años para ambos sexos; entre los hombres había un rango de 15 a 70 años (media=32.4 años, mediana=30 años y $D E=11.3$ ), y entre las mujeres, con rango de entre 21 y 45 años (media=32.6 años, mediana=35 años y $D E=7.9$ ); no se encontraron diferencias estadísticamente significativas $(p=0.7)$ entre edad y sexo. El tiempo promedio de trabajo en la empresa actual fue de 49 meses (hombres: 49; mujeres: 45). En el momento de realizar la entrevista, $85.3 \%$ (99) de los trabajadores se encontraban desempeñando labores donde se manipulaba en forma directa el plomo (soldador, tornero, fundidor, rejillador, mezclador y ensamblador).

A la totalidad de los trabajadores que participaron en el estudio se les hizo determinación de PPz en sangre, y se encontró un rango de 25 a $602 \mu \mathrm{g} / \mathrm{dl}$ (me$\mathrm{dia}=136.8 \mu \mathrm{g} / \mathrm{dl}$, mediana $=87.5 \mu \mathrm{g} / \mathrm{dl}$ y $\mathrm{DE}=116.3)$. El $60.3 \%$ (70) de los trabajadores presentó niveles de $\mathrm{PPz}$ mayores a $70 \mu \mathrm{g} / \mathrm{dl}$; éstos tenían un promedio de cuatro años de antigüedad en la empresa, $48.6 \%$ se cambiaba de ropa de trabajo una vez por semana, $61.4 \%$ lavaba la ropa en la casa y $15.7 \%$ había presentado intoxicación por plomo; el restante $39.7 \%$ (46) mostró niveles menores o iguales a $70 \mu \mathrm{g} / \mathrm{dl}$. En relación con los niveles de PbS, sólo a 81\% (94) de los trabajadores fue posible hacerles dicha determinación, la cual estableció un intervalo de 15 a $369 \mu \mathrm{g} / \mathrm{dl}$ (media $=88 \mu \mathrm{g} / \mathrm{dl}$, mediana $=66 \mu \mathrm{g} / \mathrm{dl}$ y $\mathrm{DE}=63$ ). El $84 \%$ (79) presentó niveles de plomo mayores a 38 $\mu \mathrm{g} / \mathrm{dl}$, valores definidos como de población ocupacionalmente expuesta. Dichos trabajadores habían laborado un promedio de 4.3 años en la empresa, $49.4 \%$ se cambiaba la ropa de trabajo una vez por semana, 
$60.8 \%$ lavaba la ropa de trabajo en la casa y $16.5 \%$ se había intoxicado por plomo; el 16\% (15) restante presentó niveles menores o iguales a $38 \mu \mathrm{g} / \mathrm{dl}$.

$\mathrm{Al}$ revisar los valores de PPz por sexo, los hombres mostraron un promedio de $142.1 \mu \mathrm{g} / \mathrm{dl}$ (mediana $=94 \mu \mathrm{g} / \mathrm{dl}$ y $\mathrm{DE}=118)$, y las mujeres, de $95.5 \mu \mathrm{g} / \mathrm{dl}$ (mediana $=68 \mu \mathrm{g} / \mathrm{dl}$ y $\mathrm{DE}=95.8$ ); no se encontraron diferencias estadísticamente significativas entre los niveles de PPz y el sexo ( $p=0.08)$. El nivel promedio de $\mathrm{PbS}$ en hombres fue de $92 \mu \mathrm{g} / \mathrm{dl}$ (mediana $=68 \mu \mathrm{g} / \mathrm{dl}$ y $\mathrm{DE}=65$ ), y en mujeres, de $56 \mu \mathrm{g} / \mathrm{dl}$ (mediana $=39$ $\mu \mathrm{g} / \mathrm{dl}$ y $\mathrm{DE}=43$ ), de tal forma que se encontraron diferencias estadísticamente significativas entre los niveles de $\mathrm{PbS}$ y sexo $(p=0.01)$.

Para la construcción del modelo de regresión lineal, de los 116 resultados de $\mathrm{PPz}$ se excluyeron aquellos que no tenían la determinación de $\mathrm{PbS}$ (22), y de los trabajadores que no cumplían con el requisito de tener resultados con valores por encima del punto de corte tanto para $\mathrm{PPz}(>70 \mu \mathrm{g} / \mathrm{dl})$ como para $\mathrm{PbS}(>38 \mu \mathrm{g} / \mathrm{dl})(26)$; se obtuvo así un total de 68 pares de datos. Se encontró un bajo coeficiente de correlación lineal, por lo cual, basados en reportes de la literatura, ${ }^{9,11-13,26,27}$ y con el fin de ajustar el modelo, se realizó una transformación logarítmica de los valores de PPz y se observó que el coeficiente de correlación semilogarítmica entre estos valores y los niveles de plomo fue $r=0.54$ (figura 1).

En relación con las medidas de higiene y seguridad industrial, sólo $17.2 \%$ (20) de los trabajadores se cambiaba la ropa de trabajo todos los días, $68.1 \%$ (79) lavaba la ropa en la casa y $30.2 \%$ (35) utilizaba botas de caucho. El 73.3\% (85) usaba respirador, y de éstos sólo 17.6\% (15) empleaba respirador apto para vapores

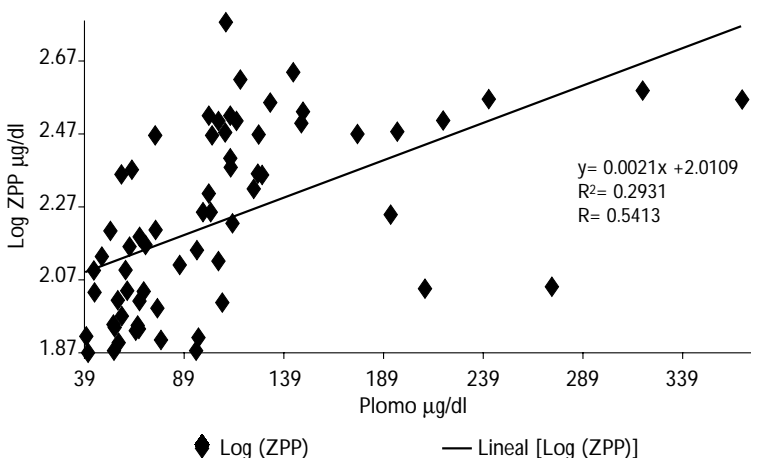

Figura 1. Regresión lineal entre los niveles de PPz y PBS de trabajadores de fábricas de baterías en BOGOTÁ, COLOMBIA, 1996 inorgánicos; del total de trabajadores que utilizaban respirador, 29.4\% (25) cambiaba el filtro una vez al mes. Respecto a hábitos como comer y fumar, se encontró que $54.3 \%$ (63) ingería alimentos y $43.9 \%$ (18) de los 41 trabajadores que fumaban lo hacían en el sitio de trabajo. El 16.3\% (19) había trabajado en otras empresas donde utilizaban plomo y todos desempeñaban labores de exposición directa. En el cuadro I se resumen algunas otras características de los trabajadores participantes en el estudio.

Se encontraron algunos signos y síntomas vagos e inespecíficos que pueden ser compatibles con exposición a plomo; los más frecuentes reportados por los trabajadores fueron cefalea $41.4 \%$ (48), irritación de la garganta e irritación de los ojos $27.6 \%$ (32), dolor abdominal $24.1 \%$ (28), visión borrosa $23.3 \%$ (27), calambres y hormigueos en miembros superiores $20.7 \%$ (24)

\section{Cuadro I \\ Medidas de Higiene y Seguridad Industrial EN LOS TRABAJADORES DE FÁBRICAS DE BATERÍAS en Bogotá, Colombia, 1996}

\begin{tabular}{llll} 
Variable & Característica & No. & $\%$ \\
Tipo de ropa & Uniforme & 90 & 77.6 \\
\hline & Traje de calle & 26 & 22.4
\end{tabular}

\begin{tabular}{llll} 
Tipo de guantes & Caucho & 60 & 51.7 \\
\hline & Cuero & 35 & 30.2 \\
\hline No los usa & 21 & 18.1
\end{tabular}

\begin{tabular}{llll} 
Tipo de respirador & Vapores inorgánicos & 15 & 12.9 \\
\hline & Tapabocas desechable & 70 & 60.4 \\
\hline No la usa & 31 & 26.7
\end{tabular}

\begin{tabular}{llll} 
Uso de calzado & Caucho & 35 & 30.2 \\
\hline & Cuero & 81 & 69.8 \\
& & & \\
Toma alimentos en el trabajo & Sí & 63 & 54.3 \\
\hline & No & 53 & 45.7
\end{tabular}

\begin{tabular}{llrr} 
Se lava las manos antes de comer & Siempre & 57 & 49.1 \\
\hline & O casionalmente & 6 & 5.2 \\
\hline N unca & 53 & 45.7
\end{tabular}

\begin{tabular}{llll} 
Se ducha al terminar la jornada & Siempre & 61 & 52.6 \\
\hline & O casionalmente & 12 & 10.3 \\
\hline N unca & 43 & 37.1
\end{tabular}

Fuente: Encuesta Laboral de losTrabajadores . Laboratorio Salud Ambiental, Instituto N acional de Salud 
y sabor metálico 19.8\% (23). De los 116 trabajadores entrevistados, $12.9 \%$ (15) había presentado intoxicación por plomo, y de éstos, $73.3 \%$ (11) había recibido tratamiento.

En el análisis estadístico se encontraron posibles asociaciones entre los niveles elevados de $\mathrm{PPz}(>70$ $\mu \mathrm{g} / \mathrm{dl}$ ) y haber desempeñado o estar desempeñando oficios de exposición directa, utilizar plomo como materia prima y cambio poco frecuente ( $\leq 1 \mathrm{vez} /$ semana) de ropa de trabajo (cuadro II). Para la sintomatología reportada, únicamente se encontró una posible asociación con irritación de los ojos (RM:3.94, IC 95\% $1.38-12.79 ; p<0.01)$. No se encontraron diferencias estadísticamente significativas entre los niveles de $\mathrm{PPz}$ $(>70 \mu \mathrm{g} / \mathrm{dl})$ y edad $(p=0.44)$, sexo $(p=0.07)$ y haber pre-

\section{Cuadro II \\ RAZONES DE MOMIOS ENTRE ALGUNAS VARIABLES Y NIVELeS EleVAdos de PPZ PResentados POR LOS TRABAJADORES DE FÁBRICAS DE BATERÍAS en Bogotá, Colombia, 1996}

Características

No. personas RM (IC 95\%) $p$

O ficio anterior en la empresa

\begin{tabular}{lrccc} 
Exposición indirecta & 14 & 1.0 & - \\
\hline Exposición directa & 102 & $4.58(1.20-21.18)$ & $<0.01$
\end{tabular}

O ficio actual en la empresa

\begin{tabular}{llcc} 
Exposición indirecta & 17 & 1.0 & - \\
\hline Exposición directa & 99 & $3.35(1.02-11.91)$ & 0.02
\end{tabular}

Utiliza plomo en su oficio

\begin{tabular}{cccc} 
No & 36 & 1.0 & - \\
\hline Sí & 80 & $7.80(2.96-21.03)$ & $<0.01$
\end{tabular}

Frecuencia de cambio de ropa

\begin{tabular}{lccc} 
Diario & 20 & 1.0 & - \\
\hline$\leq 1$ vez/semana & 96 & $3.55(1.17-11.01)$ & $<0.01$
\end{tabular}

Frecuencia con que utiliza respirador

\begin{tabular}{lccc} 
Siempre & 62 & 1.0 & - \\
\hline N unca & 54 & $1.91(0.84-4.40)$ & 0.09
\end{tabular}

Trabajó en otra empresa con plomo

\begin{tabular}{cccc} 
No & 97 & 1.0 & - \\
\hline Sí & 19 & $4.07(1.05-22.98)$ & 0.02
\end{tabular}

RM = Razón de momios (Riesgo relativo indirecto)

Fuente: Encuesta Laboral de los Trabajadores. Laboratorio Salud Ambiental, Instituto $\mathrm{N}$ acional de Salud sentado uno o más episodios de intoxicación por plomo $(p=0.9)$.

Al realizar el análisis de varianza (ANOVA), se encontraron diferencias de medias entre los niveles de PPz y algunas variables, que a su vez se pueden correlacionar con las posibles asociaciones descritas anteriormente (cuadro III). Al realizar el análisis estratificado (por el método de Mantel y Haenszel), se encontró que no existen variables que confundan los resultados debido a su intervención.

\section{Cuadro III \\ Diferencias de medias estadísticamente Significativas en ReLación Con los NiVeles de PPZ PRESENTADOS POR LOS TRABAJADORES DE FÁBRICAS de baterías en Bogotá, Colombia, 1996}

Característica Medias PPz $(\mu \mathrm{g} / \mathrm{dl}) \quad$ Estadístico f $\quad \mathrm{p}$

0 ficio anterior en la empresa

\begin{tabular}{lrrr} 
Exposición directa & 147.62 & & \\
\hline Exposición indirecta & 58.50 & 7.65 & $<0.01$
\end{tabular}

O ficio actual en la empresa

\begin{tabular}{lrrr} 
Exposición directa & 148.36 & & \\
\hline Exposición indirecta & 69.90 & 6.94 & $<0.01$
\end{tabular}

Utiliza plomo en su oficio

\begin{tabular}{lrrr} 
Sí utiliza & 170.79 & & \\
\hline N o utiliza & 61.48 & 26.85 & $<0.01$
\end{tabular}

Frecuencia de cambio de ropa

\begin{tabular}{lrrr}
$\leq 1$ vez/semana & 150.08 & & \\
\hline Diario & 73.40 & 7.61 & $<0.01$
\end{tabular}

Cómo lava la ropa

\begin{tabular}{lrrr} 
Mezclada & 53.36 & & \\
\hline No mezclada & 147.40 & 8.01 & $<0.01$
\end{tabular}

Usa guantes en el trabajo

\begin{tabular}{lrrr} 
No usa guantes & 80.63 & & \\
\hline Usa guantes & 150.77 & 7.06 & $<0.01$
\end{tabular}

Tipo de guantes

\begin{tabular}{lrrr} 
Inadecuados & 83.63 & & \\
\hline Adecuados & 186.55 & 28.00 & $<0.01$
\end{tabular}

Tipo de calzado

\begin{tabular}{llll} 
Inadecuado & 123.50 & & \\
\hline Adecuado & 167.79 & 3.62 & 0.05
\end{tabular}

Fuente: Encuesta Laboral de los Trabajadores . Laboratorio Salud A mbiental, Instituto N acional de Salud 


\section{Discusión}

La exposición al plomo se presenta como una enfermedad ocupacional y ambiental de gran importancia en salud pública en Colombia. ${ }^{14-16,18,24}$ En este estudio se encontró que $85.3 \%$ de la población estudiada realizaba actividades de exposición directa; 79 de los 94 trabajadores a los que se les realizó $\mathrm{PbS}$, presentaron niveles mayores de $38 \mu \mathrm{g} / \mathrm{dl}$ (mediana=77 $\mu \mathrm{g} / \mathrm{dl}$, $\mathrm{DE}=63)$, y 70 de los 116 trabajadores presentaron niveles de PPz mayores de $70 \mu \mathrm{g} / \mathrm{dl}$. Es interesante anotar que 11 de los 79 trabajadores que presentaron niveles de $\mathrm{PbS}$ por arriba de $38 \mu \mathrm{g} / \mathrm{dl}$ mostraron niveles de $\mathrm{PPz}$ menores de $70 \mu \mathrm{g} / \mathrm{dl}$, lo que podría indicar una reciente exposición a plomo. ${ }^{7,8,11,28}$ Los 11 trabajadores manipulaban el metal en sus procesos laborales y, para los cuales $63.6 \%$ (7) usaba como único elemento de protección respiratoria un tapabocas desechable; el restante 36.4\% (4) no usaba ningún tipo de protección.

Al realizar el análisis de varianza entre los valores de PPz y PbS por sexo, únicamente se observó diferencia significativa para PbS; esto se debió a que los hombres desarrollaban oficios de exposición directa y la mayoría de mujeres laboraban en el área administrativa donde había una exposición más baja, de forma muy variada e intermitente, al plomo durante las jornadas laborales. Esto se explica por el análisis de las variables ajustadas por edad y tiempo de trabajo en la empresa. Además, la producción en estas empresas informales es muy irregular por las características de la demanda del mercado de baterías.

En cuanto a la sintomatología presentada por los trabajadores, la posible asociación encontrada entre $\mathrm{PPz}$ e irritación de los ojos puede ser explicada por los vapores de plomo y de ácido sulfúrico que se desprenden durante el proceso de reciclaje y fabricación de baterías, y porque no emplean ningún tipo de protección para los ojos. Respecto a las diferencias de medias de algunas variables como lavar la ropa mezclada con el resto de ropa, uso y tipo de guantes y tipo de calzado, las medias de PPz fueron mayores en las condiciones óptimas en donde se esperaba que fueran lo contrario, hecho que se puede explicar por el inadecuado uso de los elementos de protección personal en trabajadores con alta exposición.

Al buscar la correlación semilogarítmica entre los valores de $\mathrm{PPz}$ y $\mathrm{PbS}$ no se encontró una buena correlación lineal $(r=0.54)$, lo que puede ser explicado debido a que se hallaron valores extremadamente altos de PPz $(602 \mu \mathrm{g} / \mathrm{dl})$, mismos que podrían indicar una prolongada y alta exposición al plomo en población ocupacionalmente expuesta; estos resultados concuerdan con los reportados en la literatura. ${ }^{11,13,27-30}$ Asimis- mo, muchos autores anotan que la falta de una fuerte correlación entre niveles de PPz y PbS podría ser explicada en términos de alta variabilidad en la cinética de absorción de plomo y formación de PPz, anemia por deficiencia de hierro, aumento de bilirrubinas, tiempo de exposición al plomo ${ }^{11,13,24,26,27,31}$ o por cambios en las condiciones de exposición, ${ }^{13}$ ya que si ésta es estable el logaritmo de PPz es linealmente relacionado con PbS. ${ }^{9,12,13,26}$ En este estudio se pudo observar la poca permanencia de los trabajadores en un oficio, debido a que estos desempeñan simultáneamente diferentes actividades durante los procesos de reciclaje y fabricación de baterías.

Con base en los resultados mencionados se realizó una comparación de los datos de $\mathrm{PPz}$ como prueba tamiz con PbS y con el fin de determinar las características del método; se obtuvo una sensibilidad de 86.1\% (IC 95\% 76.0-92.5), una especificidad de 93.3\% (IC 95\% 66.0-99.7) y una eficacia de $87.2 \%$, datos similares a los reportados en estudios realizados en Colombia ${ }^{15,18,23,24}$ y en el ámbito internacional. ${ }^{24,26,30} \mathrm{Ba}-$ sados en la especificidad de la prueba, se observó que un nivel para PPz de $70 \mu \mathrm{g} / \mathrm{dl}$ o menos (con un $95 \%$ de probabilidad) es un buen valor límite por debajo del cual generalmente se encuentran valores de plomo de $38 \mu \mathrm{g} / \mathrm{dl}$ o menos. Por lo anterior, la prueba de $\mathrm{PPz}$ puede servir como prueba tamiz en actividades de vigilancia epidemiológica para identificar con gran confiabilidad individuos "sanos". Igualmente, es posible monitorear un gran número de trabajadores por ser una prueba simple, rápida y de bajo costo. 1,4,8-12,19,26,30,32,33

En Colombia clásicamente se ha recomendado la medición de los niveles de $\mathrm{PbS}$ como el método de seguimiento confiable para los trabajadores que manipulan el metal; sin embargo, la experiencia ha demostrado que dicha prueba no ha tenido el uso masivo que se requiere, entre otros factores por su alto costo $(75 \%$ más que la $\mathrm{PPz}){ }_{i}^{24}$ por ello en este estudio se exploró la posible utilidad de la $\mathrm{PPz}$ como prueba tamiz en programas de vigilancia epidemiológica para trabajadores que se exponen al plomo. No obstante, la determinación de PPz en sangre no puede ser usada como única determinación para indagar casos de absorción ocupacional de plomo, ni como un indicador discriminativo, especialmente en aquellos casos donde la absorción es muy baja y reciente, ya que niveles elevados de PPz también resultan en anemias por deficiencia de hierro. Por lo tanto, se infiere que no se pueden predecir niveles de PbS a partir de valores de PPz. Es importante anotar que altos valores individuales pueden o no ser el resultado de una exposición a plomo, pero la medida directa de la $\mathrm{PPz}$ 
puede ser un índice útil y un buen indicador diagnóstico de intoxicación por plomo en trabajadores industriales con exposición a este metal. ${ }^{12,26,30}$

El estudio tuvo limitaciones en relación con la historia de exposición laboral de los trabajadores, la cual no fue posible obtener ya que ninguna empresa contaba con registros sobre monitoreos biológicos y ambientales. Tampoco se obtuvo información sobre exposiciones extralaborales a plomo debido a que el instrumento diseñado no contenía este tipo de variables.

Estos resultados, aunados a la ausencia de programas de capacitación y campañas de sensibilización sobre los efectos que en la salud desencadena la exposición a este metal, pueden indicar que estos trabajadores no son conscientes del peligro al que están expuestos, lo cual se aprecia en las inapropiadas medidas de higiene y seguridad, como cambio poco frecuente de ropa de trabajo, alto porcentaje de trabajadores que lavaban la ropa de trabajo en la casa, bajo uso de respiradores adecuados para protegerse del plomo inorgánico, poco y mal uso de guantes y botas de caucho, y alto porcentaje de trabajadores que ingerían alimentos y fumaban en el sitio de trabajo. Igualmente, estas microempresas familiares pueden constituir fuentes de intensa exposición a plomo en el medio ambiente laboral, lo que implica un problema no sólo para los trabajadores sino para sus familias, debido a que éstos pueden transportar partículas de plomo en sus ropas, puesto que no cuentan con lugares apropiados para ducharse y cambiarse de ropa de trabajo, condiciones que podrían disminuir este riesgo. $3,32,34$

La solución del problema de intoxicación por plomo en nuestro medio puede ser un poco difícil, considerando que la mayoría de microempresas del sector informal no están registradas en los organismos gubernamentales; no cuentan con los recursos para afiliarse a una Aseguradora de Riesgos Profesionales (ARP) ni con un adecuado Régimen Subsidiado de Salud (ARS); tampoco se cuenta con un Plan de Atención Básica (PAB) que evalúe el componente ocupacional del sector informal, lo que complica este problema de salud pública. Se hace necesario un esfuerzo conjunto de organismos de salud, entidades de salud pública, protección ambiental, organismos territoriales de salud, secretarías de salud y entidades educativas con el fin de desarrollar programas de vigilancia para los trabajadores del sector informal de la economía, lo cual puede lograrse diseñando tecnología de producción más limpia y de menor costo, realizando estudios de monitoreo ambiental y facilitando el acceso de los trabajadores y sus familias a los servicios de salud, con el propósito de prevenir y controlar los casos de intoxicación. Finalmente, es importante incluir en el PAB que las empresas y los trabajadores que manipulan el plomo se capaciten en el adecuado uso y manejo de los elementos de protección personal, y que sigan las medidas de higiene y seguridad industrial.

Es indispensable que se realicen estudios analíticos para la determinación de valores de referencia con el propósito de ajustar la normatividad en Colombia, cuyo objetivo sea el de proteger la salud de los trabajadores y que en el ámbito ocupacional los valores de referencia para plomo en sangre sean los más bajos posible.

\section{A gradecimientos}

Los autores agradecen a los trabajadores y gerentes de las fábricas de baterías participantes en el estudio. Igualmente, a los dos técnicos de la unidad de Espectrofotometría del Laboratorio Salud Ambiental del Instituto Nacional de Salud, Bogotá, Colombia, por el apoyo brindado en el análisis de plomo en sangre y la determinación de la zinc protoporfirina en sangre. De igual forma, el agradecimiento a la doctora Nelcy Rodríguez (Bioestadística) por su asesoría.

\section{Referencias}

1. Corey G, Galvao L. Plomo. Serie Vigilancia N 0. 8. México, D.F.: Editorial Clemente A guilar, 1989:1-45.

2. Dreisbach R. Manual de envenenamientos. 3a. edición. México, D.F.: El Manual Moderno, 1978:190-196.

3. $N$ ational A cademy of Sciences. Biologic effects of atmospheric pollutants. Lead. Airbone lead in perspective. W ashington, D.C.: N AS, 1972: 126-132.

4. O rganización Panamericana de la Salud/O rganización Mundial de la Salud. Plomo. México, D.F.: Servicio de Publicaciones y Documentación de la O PS/O MS (Criterios de Salud Ambiental, núm. 3), 1979:3-319.

5. G orey RA.Toxic effects of metals. Lead. En: Cassarett y D oull's. Toxicolo gy.The basic science of poisons. 4a. edición. N uevaYork: Pergamon Press/ Maxwell Macmillan Publishing Corporation, 1991:639-646.

6. Sanín LH, González T, Romieu I, Hernández M. A cumulación de plomo en hueso y sus efectos en la salud. Salud Publica Mex 1998:40:359-368.

7. A guilar $G$, Piacitelli GM, Juárez CA, Vázquez JH, Hu H, Hernández M. Exposición ocupacional a plomo inorgánico en una imprenta de la ciudad de México. Salud Publica Mex 1999;41:42-54.

8. Hesley KL,W imbish GH. Blood lead and zinc protoporphyrin in lead industry workers. Am Ind Hyg Assoc J 1981;42:42-46.

9. Kononen DW. First-year changes in blood lead and zinc protoporphyrin levels within two groups of occupational lead workers. Am Ind $\mathrm{HygAssoc}$ J 1991:52:177-182.

10. Labbé RF. Clinical utility of zinc protoporphyrin. Clin Chem 1992; 38:2167-2168.

11. Suga RS, Fischinger AJ, Knoch FW. Establishment of normal values in adults for zinc protoporphyrin (ZPP) using hematofluorometer: correlation with normal blood lead values. Am Ind HygAssoc J 1981;42:637-642. 
12. Grunder Fl, Moffitt AE. Evaluation of zinc protoporphyrin in an occupational environment. Am Ind Hyg Assoc I 1979;40:686-694.

13. Hryhorckzuk DO, Hogan MM, Mallin K, Hessl SM, O rris P.The fall of zinc protoporphyrin levels in workers treated for chronic lead intoxication. J 0 ccup Med 1985;27:816-820.

14. Andi-Colombia. Implicaciones de la salud ocupacional en la industria moderna. Rev Andi 1984;9:35-37.

15. Podlesky E, 0 rtiz J. Estudio comparativo de plomo en sangre y zinc protoporfirina (ZPP) como método de tamizaje aplicable a actividades de vigilancia epidemiológica de personas expuestas al plomo. Biomedica 1990;10 Suppl. 50.

16.Varona M. Reporte de evaluación monográfica de un problema de salud pública: panorama epidemiológico de la exposición ocupacional y ambiental al plomo en Colombia 1987-1993. Santa Fe de Bogotá: Instituto N acional de Salud, 1995:74-98.

17. Podlesky E,Varona M, O rtiz J,Villamil G, Q uiñonez H,Alfonso G. Toma de muestras ambientales y biológicas para análisis toxicológicos. Manual de procedimientos. Santa Fe de Bogotá, Instituto N acional de Salud, 1990:9-14.

18. Celis JC, Medina LJ. Determinación de zinc protoporfirina como índice de la contaminación con plomo. Bogotá: Universidad de los Andes, 1990:46-78.

19. Piomelli S.A micromethod for free erythrocyte porphyrins:The FEP test. J Lab Clin Med 1973;6:932-940.

20. $\mathrm{N}$ ational Institute for $\mathrm{O}$ ccupational Safety and Health. Manual of analytical methods. Lead in blood and urine. Method 8003. 3a. edición. Cincinnati: Eller PM, 1984;1:8003-1-8003-4.

21. Podlesky E, 0 rtiz J,Villamil G. Determinación de trazas de metales en muestras biológicas y ambientales. Manual de procedimientos. Santa Fe de Bogotá: Instituto N acional de Salud, 1992;39-42.

22. Ministerio de Salud. Dirección de Promoción y Prevención. Guía de atención integral de enfermedades profesionales: plomo. Santa Fe de Bogotá: Ministerio de Salud, 1997:1-47.

23. U ribe $C$, G utiérrez M, Patiño N, Uribe M, Martínez $0, G$ root $H$ et al. Prevalencia de intoxicación por plomo en trabajadores de fábricas de baterías y estudio comparativo entre una prueba de tamizaje y una "prueba de oro". D ocumento Técnico. Santa Fe de Bogotá: Instituto de Seguros Sociales, Clínica de Toxicología LTDA. 1996:1-64.
24. Cuevas H, O rtiz J, De La Hoz F, Raad J.Vigilancia de los niveles de zinc protoporfirina (ZPP) y plomo en sangre en trabajadores de una imprenta en Santa Fe de Bogotá. Biomedica 1997;17:56-61.

25. Ministerio de Trabajo y Seguridad Social-Dirección General de la Seguridad Social-División de Salud 0 cupacional. Compendio de normas legales sobre salud ocupacional. Ley 9a. Título 30. Salud 0 cupacional. Resolución 02400 Estatuto de Seguridad Industrial. Capítulo 80. Artículo 154 de las concentraciones máximas permisibles. Santa Fe de Bogotá: Instituto N acional de Salud, 1979.

26. Z wennis W C, Franssen Ach, W ijnans MJ. U se of zinc protoporphyrin in screening individuals for exposure to lead. Clin Chem 1990;36: 1456-1459.

27. Simmonds PL, Luckhurst CL,W oods JS. Q uantitative evaluation of heme biosynthetic pathway parameters as biomarkers of low-level lead exposure in rats. J Toxicol Environ Health 1995;44:351-367.

28. Turk DS, Schonfeld DJ, C ullen M, Rainey P. Sensitivity of erythrocyte protoporphyrin as a screening test for lead poisoning. $N$ Engl J Med 1992;326:137-138.

29. Froom P, Kristal-Boneh E, Benbassat J,A shkanazi R, Ribak J. Predictive value of determinations of zinc protoporphyrin for increased blood lead concentrations. Clin Chem 1998;44:1283-1288.

30.Zhang J. Investigation and evaluation of zinc protoporphyrin as a diagnostic indicator in lead intoxication. Am J Ind Med 1993;24:707-712.

31. Schonfeld DJ, C ullen MR, Rainey PM, BergAT, Brwn DR, Hogan JC et al. Screening for lead poisoning in an urban pediatric clinic using samples obtained by fingerstick. Pediatrics 1994;94:174-179.

32. Ritchter E, Fischbein A. Lead poisoning: II. Biological standards for occupational lead exposure-W here do we stand now? Isr J Med Sci 1992; 28:572-577

33. Joselow MM, Flores J.Aplication of the zinc protoporphyrin (ZPP) test as a monitor of occupational exposure to lead. Am Ind Hyg Assoc J 1977;38:63-66.

34. Gittleman JL, Engelgau MM, Shaw J,W ille KK, Seligman PJ. Lead poisoning among battery reclamation workers in Alabama. JO M 1994;36: 526-532. 